\title{
Limited value of ultrasound assessment in patients with poor outcome after carpal tunnel release surgery
}

\author{
A Naranjo ${ }^{1}$, S 0jeda ${ }^{1}$, I Rúa-Figueroa' 0 Garcia-Duque ${ }^{2}$, J Fernández-Palacios ${ }^{2}$, L Carmona ${ }^{3}$ \\ Departments of ${ }^{1}$ Rheumatology and ${ }^{2}$ Plastic and Reconstructive Surgery, Doctor Negrin University Hospital of Gran Canaria, The University \\ of Las Palmas, Gran Canaria, and ${ }^{3}$ Research Unit, Spanish Foundation of Rheumatology, Madrid, Spain
}

\begin{abstract}
Objective: To determine the value of ultrasonography in the assessment of patients with idiopathic carpal tunnel syndrome (CTS) and poor outcome after carpal tunnel release.

Methods: A total of 88 consecutive patients with CTS (104 hands) underwent open surgical release of the median nerve. Ultrasound (US) examination was performed blind to any patient's data. The median nerve area at tunnel inlet and outlet, the retinaculum distance, and the flattening ratio were measured. The main outcome variable was the patient's overall satisfaction using a five-point Likert scale $(1=$ worse, $2=$ no change, $3=$ slightly better, $4=$ much better, $5=$ cured $)$ at 3 months postoperatively. Pre- and postoperative ultrasonographic findings in relation to clinical outcome were analysed. Results: Improvement (scores 4 or 5 on the Likert scale) was recorded in 75 hands (72\%). After carpal tunnel release, the cross-sectional area at tunnel inlet decreased from a mean of 14.2 to $13.3 \mathrm{~mm}^{2}$ in the group with clinical improvement and also from a mean of 12.5 to $11.6 \mathrm{~mm}^{2}$ in the group with no change or slight improvement. No significant changes in the cross-sectional area at tunnel outlet, retinaculum distance, and flattening ratio were observed.

Conclusion: Reduction of the median nerve cross-sectional area at tunnel inlet at 3 months after carpal tunnel release was similar in patients reporting cure or great improvement and in those with slight or no improvement. Ultrasonography is of limited value in assessment of patients with poor outcome after median nerve release.
\end{abstract}

Some patients with unsatisfactory results after carpal tunnel release present a difficult diagnostic problem because most of them continue to show abnormal findings at postsurgical electrophysiological evaluation $(1,2)$, even in those who are asymptomatic. There are limited data evaluating the clinical use of ultrasonography in dissatisfied patients after median nerve release. Therefore, a prospective observational study in a large and homogeneous group of patients with carpal tunnel syndrome (CTS) was performed to determine the value of ultrasonography in the assessment of those patients with poor outcome after surgery.

\section{Material and methods}

Between July 2005 and December 2006, all consecutive adult patients with suspected CTS referred to our institution and all of whom who underwent CTS surgery were selected for the study. The study protocol was approved

\footnotetext{
Antonio Naranjo, Department of Rheumatology, Doctor Negrin University Hospital of Gran Canaria, Barranco de la Ballena s/n, E-35010 Las Palmas de Gran Canaria Spain.

E-mail: anarher@gobiernodecanarias.org

Accepted 8 February 2010
}

by the Institutional Review Board and all participants gave their written informed consent.

Suspected idiopathic CTS was defined by sensory symptoms over the distribution territory of the median nerve regardless of the results of Tinel nerve percussion and Phalen's manoeuvre. Sensory symptoms included hypoaesthesia, burning pain, tingling, or numbness aggravated by sustained positions and relief by shaking or moving the hands, sleep disruption by symptoms, and daily complaints for at least 3 months. Patients were excluded if they had already undergone surgery, had suffered traumatic injuries at the target wrist, had received injections, presented ganglions or tenosynovitis, as were those with hypothyroidism, acromegaly, polyneuropathy, radiculopathy, fibromyalgia, diabetic neuropathy, rheumatoid arthritis, or crystal arthritis. Pregnant women were also excluded.

Patients were initially assessed by a single rheumatologist (SO) who obtained the clinical history and performed the physical examination. Patients completed the Spanish validated version of the Boston-Levine CTS health-related quality of life questionnaire (3). The total score is given in two scales (range 1-5), symptoms and function, according to the severity of symptoms and the difficulty of performing a given activity, in an ascending 
manner. If both hands were involved, patients fulfilled two questionnaires, one for each hand.

\section{Ultrasonography}

Ultrasound (US) examinations were performed by an experienced rheumatologist (AN) who was unaware of the medical history and the results of nerve conduction studies (NCS). All patients underwent high-resolution real-time sonography of the carpal tunnel using a General Electric Logic 5 Pro US machine and a $12 \mathrm{MHz}$ linear array transducer before surgery and 3 months after carpal tunnel release. The carpal tunnel inlet was defined as the proximal margin of the flexor retinaculum between the scaphoid tubercle and the pisiform bone, and the tunnel outlet was the distal margin of the flexor retinaculum between the trapezium bone and the hook of the hamate bone. Measurements were taken for the median nerve at the carpal tunnel inlet proximally and at the carpal tunnel outlet distally by direct tracing with electronic callipers excluding the echogenic rim surrounding the nerve. We performed assessments twice and recorded the arithmetic mean of two assessments. The flattening ratio, defined as the ratio of the major axis of the median nerve to its minor axis, was also assessed at the level of the hamate bone. The maximum height or bowing of the retinaculum was measured above a line subtended between the radial attachment in the trapezium and the ulnar attachment in the hamate and the top of the flexor retinaculum.

\section{NCS}

NCS were performed under the guidance of two neurologists following the American Academy of Neurology protocol (4). CTS severity was defined as normal, mild, moderate, or severe.

\section{Surgery}

Patients with typical symptoms and daily complaints for at least 3 months were candidates for median nerve release, including patients with normal CNS, except those with a median nerve cross-sectional area at the tunnel inlet $<11 \mathrm{~mm}^{2}$. The open technique under regional anaesthesia with tourniquet control was performed in all cases. If both hands were involved, the most symptomatic was operated on first.

\section{Outcome measures}

The main outcome variable was the patient's overall satisfaction on a five-point Likert scale ( 1 = worse, $2=$ no change, 3 = slightly better, $4=$ much better, 5 = cured) at 3 months postoperatively. Success was defined as scores
4 or 5 . Outcome assessments were performed by a single examiner blinded to the NCS and US data.

\section{Statistical analysis}

We studied the distribution of pre- and postoperative clinic and US variables in all patients and in those who responded and who did not respond to surgery at 3 months. Differences before and after surgery between groups with and without improvement were tested by the Student's t test. Pearson's correlation coefficient was used to analyse the relationship between US and Boston questionnaires changes pre/postsurgery.

\section{Results}

A total of 88 patients (15 men and 73 women) with a mean (SD) age of 50 (11) years fulfilled the inclusion criteria. In 16 patients (18.2\%), both hands were affected. The total number of hands evaluated was 104 . The mean (SD) duration of clinical symptoms was 30 (31) months. Preoperatively, CTS severity by NCS criteria was classified as severe in 38 hands, moderate in 45 , and mild in 10 . In the remaining 11 cases, no electrophysiological evidence of CTS was documented. The cross-sectional area of the median nerve at tunnel inlet was $>11 \mathrm{~mm}^{2}$ in $87(83.6 \%)$ cases.

The results at 3 months are shown in Table 1 . With regard to the outcome variable, overall satisfaction was rated as cured in 20 cases, much better in 55, slightly better in 20 , no change in 3 , and worse in 6 . When the categories of cured and much better were grouped together (n $=75$ ) and compared with slightly better, no change, and worse $(\mathrm{n}=29)$, the mean cross-sectional area at tunnel inlet preoperatively was significantly higher in the clinical improvement group than in the group without clinical improvement (14.2 vs. $12.5 \mathrm{~mm}^{2}, \mathrm{p}=0.03$ ). However, when the pre- and postoperative mean cross-sectional areas of the median nerve at tunnel inlet were compared, significant decreases were observed in both categories. It should be noted that of 20 patients with more than $2 \mathrm{~mm}^{2}$ reduction in the median nerve area at tunnel inlet, 12 patients showed a marked improvement and eight a slight or no improvement. No correlation was found between changes in the Boston questionnaire and changes in US cross-sectional area $(r=-0.05)$. Differences in other US measurements, including cross-sectional area at tunnel outlet, flattening ratio, and retinaculum distance, were not found. Moreover, the US studies did not show any case of synovitis or flexor tenosynovitis.

\section{Discussion}

Postoperative magnetic resonance imaging (MRI) findings CTS include division of the flexor retinaculum, changes in nerve configuration with an increased cross-sectional area, and reduced flattening ratios, as well as restoration 

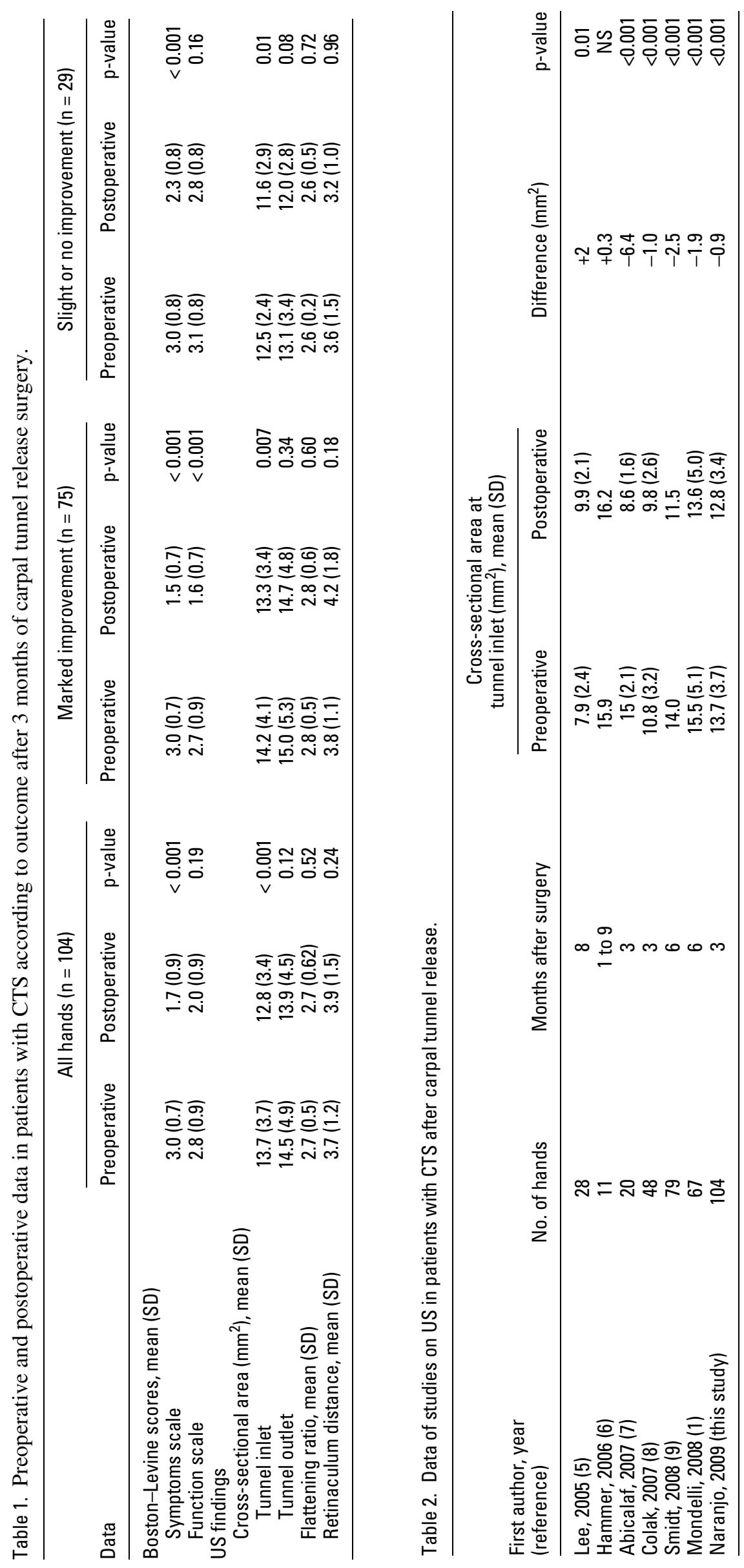
of the $\mathrm{T}$ signal intensity in the distal carpal tunnel (2). However, a clear relationship between the changes found on MRI and clinical improvement after surgery was not observed (2).

In patients with unsatisfactory results after carpal tunnel release, US is a rapid, safe, and inexpensive technique that can be useful to demonstrate incomplete division of the flexor retinaculum or a mass effect due to flexor tenosynovitis or a ganglion or prominent postsurgical scars. Several studies of US examination after median nerve release have been reported $(1,5-9)$ (Table 2). In most of these a significant decrease was recorded in the cross-sectional area of the median nerve at the tunnel inlet ranging between 1 and $3 \mathrm{~mm}^{2}$. A reduction in oedema may not be sufficient to leave the nerve at a normal size, which may account for the median nerve crosssectional areas $>11 \mathrm{~mm}^{2}$ seen postoperatively in many patients (69\% in our series). However, the clinical significance of the reduction of the cross-sectional area after median nerve release is unclear. The study of Mondelli et al (1) found a correlation between the degree of reduction of the cross-sectional area after surgery and improvement of symptoms and results of NCS. In a recent study by our group, the cross-sectional area of the median nerve was the only diagnostic feature with predictive value of a favourable surgical outcome at 3 months, in comparison with physical manoeuvres and CNS (10). By contrast, in the present study, an association between reduction of the cross-sectional area of the median nerve after operation and clinical improvement was not documented. Regarding the area of the nerve at the tunnel outlet, data reported in the literature are controversial. In the present series, no significant changes were observed, although in other studies either an increase $(1,5)$ or a decrease in the cross-sectional area (8) has been reported. Some US studies have shown normalization or reduction of the flattening ratio and an increase in the retinaculum distance after carpal tunnel surgery $(5,11)$, although we did not observe significant changes.

In this study we chosen $11 \mathrm{~mm}^{2}$ as the cutoff because in a previous study by our group we confirmed the hypothesis that improvement after surgery was independent of whether patients were selected by abnormal CNS or by US $>11 \mathrm{~mm}^{2}$ (10). One of the limitation of the present study may be the short period of assessment of only 3 months after surgery.
We conclude that ultrasonography has a limited value in the assessment of patients with unsatisfactory surgical results after carpal tunnel release.

\section{Acknowledgements}

We thank J. C. Quevedo, C. Erausquin, F. Francisco, C. RodríguezLozano, V. Araña, and D. Mendoza for their collaboration in the recruitment of patients, and M. Pulido for editorial assistance.

\section{References}

1. Mondelli M, Filippou G, Aretini A, Frediani B, Reale F. Ultrasonography before and after surgery in carpal tunnel syndrome and relationship with clinical and electrophysiological findings. A new outcome predictor? Scand J Rheumatol 2008;37:219-24.

2. Cudlip SA, Howe FA, Clifton A, Schwartz MS, Bell BA. Magnetic resonance neurography studies of the median nerve before and after carpal tunnel decompression. J Neurosurg 2002;96: 1046-51.

3. Rosales RS, Delgado EB, Diez de la Lastra-Bosch I. Evaluation of the Spanish version of the DASH and CTS health-related qualityof-life instruments: cross-cultural adaptation process and reliability. J Hand Surg Am 2002;27:334-43.

4. American Academy of Neurology, American Association of Electrodiagnostic Medicine, and American Academy of Physical Medicine and Rehabilitation. Practice parameter for electrodiagnostic studies in carpal tunnel syndrome (Summary statement). Neurology 1993;43:2404-5.

5. Lee CH, Kim TK, Yoon ES, Dhong ES. Postoperative morphologic analysis of carpal tunnel syndrome using high-resolution ultrasonography. Ann Plast Surg 2005;54:143-6.

6. Hammer HB, Hovden IA, Haavardsholm EA, Kvien TK. Ultrasonography shows increased cross-sectional area of the median nerve in patients with arthritis and carpal tunnel syndrome. Rheumatology (Oxford) 2006;45:584-8.

7. Abicalaf CA, de Barros N, Sernik RA, Pimentel BF, Braga-Baiak A, Braga L, et al. Ultrasound evaluation of patients with carpal tunnel syndrome before and after endoscopic release of the transverse carpal ligament. Clin Radiol 2007;62:891-4.

8. Colak A, Kutlay M, Pekkafali Z, Saraçoglu M, Demircan N, Simşek $\mathrm{H}$, et al. Use of sonography in carpal tunnel syndrome surgery. A prospective study. Neurol Med Chir (Tokyo) 2007;47:109-15.

9. Smidt MH, Visser LH. Carpal tunnel syndrome: clinical and sonographic follow-up after surgery. Muscle Nerve 2008;38:987-91.

10. Naranjo A, Ojeda S, Araña V, Baeta P, Fernández-Palacios J, Garcia-Duque $\mathrm{O}$, et al. Usefulness of clinical findings, nerve conduction studies and ultrasonography to predict response to surgical release in idiopathic carpal tunnel syndrome. Clin Exp Rheumatol 2009; 27:786-93.

11. El-Karabaty H, Hetzel A, Galla TJ, Horch RE, Lücking CH, Glocker FX. The effect of carpal tunnel release on median nerve flattening and nerve conduction. Clin Neurophysiol 2005;45:223-7. 